\title{
Quantum gravity corrections to the standard model Higgs in Einstein and $R^{2}$ gravity
}

\author{
Yugo Abe, Masaatsu Horikoshi \\ Department of Physics, Shinshu University, Asahi3-1-1, Matsumoto, Nagano 390-8621, Japan \\ Takeo Inami \\ Department of Physics, National Taiwan University, Taipei 10617, Taiwan, R.O.C. \\ Theoretical Research Division, Nishina Center, RIKEN, Wako 351-0198, Japan
}

\begin{abstract}
We evaluate quantum gravity corrections to the standard model Higgs potential $V(\phi)$ a la Coleman-Weinberg and examine the stability question of $V(\phi)$ at scales of Planck mass $M_{\mathrm{Pl}}$. We compute the gravity one-loop corrections by using the momentum cut-off in Einstein gravity. The gravity corrections affect the potential in a significant manner for the value of $\Lambda=(1-3) M_{\mathrm{Pl}}$. In view of reducing the UV cut-off dependence we also make a similar study in the $R^{2}$ gravity.
\end{abstract}

Keywords: Higgs potential, Coleman-Weinberg, Planck scales, cut off method, $R^{2}$ gravity

\section{Introduction}

It is curious that the mass of the Higgs boson $m_{\mathrm{H}}(=125.09 \pm 0.24 \mathrm{GeV})$, which has recently been discovered at LHC, lies far outside of the mass bound derived from the one-loop radiative corrections $\frac{1.2}{2}$. This bound arises from the stability condition on the Higgs quartic coupling $\lambda$, i.e. $\lambda(\mu)>0$. The large two-loop corrections come into play and the renormalization group (RG) flow of $\lambda$ changes drastically. The flow is also tangled with the top quark mass $m_{\mathrm{t}}$. Some finetuning of the parameters, especially that of $m_{\mathrm{t}}$, yield the $\lambda$ barely in accord with the boundary values of the stability bound extended to the scales of Planck mass $M_{\mathrm{Pl}} \cdot \underline{\underline{3}} \underline{\underline{5}}$ It implies an interesting possibility that the standard model may be the correct unification theory all the way up to the Planck scales $M_{\mathrm{Pl}} \cdot \underline{\underline{6}} \underline{\underline{8}}$ This scenario is consistent with the so far vain results of the susy particle search at the LHC experiment.

It is a common belief that quantum gravity effects should manifest itself at Planck scales, irrespectively of the correct quantum theory of gravity. Hence it is a very urgent problem to study whether incorporating quantum gravity corrections may change drastically the RG analyses with the matter loop corrections alone. ${ }^{9}-11$

In this paper, we calculate the gravity loop corrections to the Higgs potential $V(\phi)$ and examine the property of the Higgs potential at Planck scales $M_{\mathrm{Pl}}$. In the Einstein gravity, we have found that there is a significant difference between the Higgs potential with and without gravity corrections. In the previous works,,$\underline{9,10}$ the gravity loop corrections to the $\phi^{6}$ and $\phi^{8}$ terms are studied. We consider all such corrections on the RG analysis of $V(\phi)$. This talk contains a brief summary 
of our recent paper. $\underline{\underline{12}}$

\section{Gravitational Coleman-Weinberg corrections}

In the standard model (SM), one- and two-loop contributions to the bare Higgs mass and the bare couplings have been studied in details. $\cdot \underline{\underline{3}} \underline{\underline{5}}$ We take into account the gravity one-loop effects in addition to the matter loop effects and will see how two contributions compete in the energy region of Planck mass scales $M_{\mathrm{Pl}}$.

We derive the Higgs effective potential $V(\phi)$ in the Einstein's gravity theory, by extending the Coleman-Weinberg mechanism $\frac{13}{13}$ to gravity loops ${ }^{9}, 10,14$. We begin by writing the action for the Higgs field $H$ and the metric $g_{\mu \nu}$

$$
S=\int d^{4} x \sqrt{-g}\left[\frac{2}{\kappa^{2}} R+g^{\mu \nu}\left(\partial_{\mu} H\right)^{\dagger}\left(\partial_{\nu} H\right)+m^{2} H^{\dagger} H-\lambda\left(H^{\dagger} H\right)^{2}+\cdots\right] .
$$

where $\kappa:=\sqrt{32 \pi G}=\sqrt{32 \pi} M_{\mathrm{Pl}}^{-1}\left(M_{\mathrm{Pl}}=1.22 \times 10^{19} \mathrm{GeV}\right)$. The ellipses show the terms of gauge and fermion fields. Expanding the fields around background fields as $H^{\dagger}=1 / \sqrt{2}\left(\sigma_{1}-i \pi_{1}, \phi+\sigma_{2}-i \pi_{2}\right)$ and $g_{\mu \nu}=\eta_{\mu \nu}+\kappa h_{\mu \nu}$, we evaluate the gravity corrections for the tree level Higgs potential

$$
V_{\text {tree }}=-\frac{1}{2} m^{2} \phi^{2}+\frac{1}{4} \lambda \phi^{4} .
$$

We take the de Donder gauge fixing term $\mathcal{L}_{\text {gf }}$. It is given in the Minkowski background by

$$
\mathcal{L}_{\mathrm{gf}}=-\eta_{\alpha \beta}\left(\eta^{\mu e} \eta^{\nu \alpha}-\frac{1}{2} \eta^{\mu \nu} \eta^{e \alpha}\right)\left(\eta^{\rho f} \eta^{\sigma \beta}-\frac{1}{2} \eta^{\rho \sigma} \eta^{f \beta}\right) h_{\mu \nu, e} h_{\rho \sigma, f} .
$$

The Higgs and graviton 1-loop corrections to $V_{\text {tree }}(\phi)$ are obtained in the cut-off method. $\underline{9}, 10,14$

$$
\begin{aligned}
\delta V_{\mathrm{eff}}= & \frac{3}{64 \pi^{2}}\left(-m^{2}+\lambda \phi^{2}\right)^{2}\left(\ln \frac{-m^{2}+\lambda \phi^{2}}{\Lambda^{2}}-\frac{3}{2}\right) \\
& +\frac{9}{256 \pi^{2}} \kappa^{4}\left(-\frac{1}{2} m^{2} \phi^{2}+\frac{1}{4} \lambda \phi^{4}\right)^{2}\left(\ln \left(\frac{\kappa^{2}}{2} \frac{-\frac{1}{2} m^{2} \phi^{2}+\frac{1}{4} \lambda \phi^{4}}{\Lambda^{2}}\right)-\frac{3}{2}\right) \\
& +\sum_{i= \pm} \frac{C_{i}^{2}}{64 \pi^{2}}\left(\ln \frac{C_{i}}{\Lambda^{2}}-\frac{3}{2}\right),
\end{aligned}
$$

where $C_{ \pm}$is

$$
C_{ \pm}=\frac{1}{2}\left[m_{C}^{2}-m_{A}^{2} \pm \sqrt{\left(m_{C}^{2}+m_{A}^{2}\right)^{2}-16 m_{B}^{4}}\right] .
$$

Here $m_{A}^{2}=\frac{\kappa^{2}}{2}\left(-\frac{m^{2}}{2} \phi^{2}+\frac{\lambda}{4} \phi^{4}\right), m_{B}^{2}=\frac{\kappa}{2}\left(-m^{2} \phi+\lambda \phi^{3}\right)$ and $m_{C}^{2}=-m^{2}+3 \lambda \phi^{2}$.

The second and third terms in (4) are due to the graviton loops, which are suppressed by Planck mass at electro-weak scales. Gravity corrections give rise to logarithmic divergent terms of $\phi^{6}$ and $\phi^{8}$. Such higher power terms are not 
significant at usual energies, but they may become significant at high energy scales, i.e. $M_{\mathrm{Pl}}$.

The $\phi^{2}$ and $\phi^{4}$ terms may be renormalized in the usual way. Expanding the third term $\sum_{i= \pm} C_{i}^{2}=m_{C}^{4}+m_{A}^{4}-8 m_{B}^{4}$, the counter terms (CT) are found as

$$
\begin{aligned}
\delta V_{\mathrm{CT}}= & \frac{3}{64 \pi^{2}}\left(-m^{2}+\lambda \phi^{2}\right)^{2} \ln \left(\frac{\Lambda^{2}}{\mu^{2}}\right)+\frac{1}{64 \pi^{2}}\left(-m^{2}+3 \lambda \phi^{2}\right)^{2} \ln \left(\frac{\Lambda^{2}}{\mu^{2}}\right) \\
& -\frac{\kappa^{2}}{32 \pi^{2}}\left(m^{4} \phi^{2}-2 \lambda m^{2} \phi^{4}\right) \ln \left(\frac{\Lambda^{2}}{\mu^{2}}\right)+\frac{5 \kappa^{4}}{512 \pi^{2}} m^{4} \phi^{4} \ln \left(\frac{\Lambda^{2}}{\mu^{2}}\right) .
\end{aligned}
$$

We add these gravitational 1-loop corrections to the tree level potential

$$
V_{\mathrm{eff}}=V_{\mathrm{tree}}+\delta V_{\mathrm{eff}}+\delta V_{\mathrm{CT}}
$$

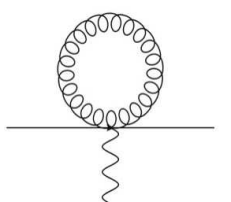

$a$

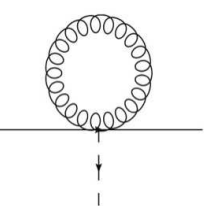

b.1

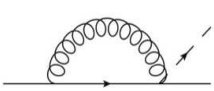

6.2

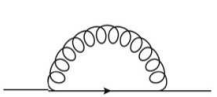

c. 1

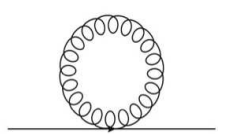

c.2

Fig. 1. Gravitational 1-loop diagrams for gauge couplings (a), Yukawa coupling (b) and anomalous dimension of fermion (c).

The Higgs potential including the loop corrections can easily be obtained by use of RG. We already know the $\beta$ functions in SM to the 2-loop order. 3.15

Some graviton loop corrections have recently been computed. $.^{9}-11$ We have further calculated gravity corrections to other coupling constants, i.e. gauge and Yukawa couplings, as shown in Fig1, $\beta$ functions and anomalous dimensions due to the gravity corrections are

$$
\begin{aligned}
& \beta_{m^{2} g}=\frac{5 \kappa^{2} m^{2}}{16 \pi^{2}} \mu^{2}-\frac{\kappa^{2} m^{4}}{8 \pi^{2}}, \beta_{\lambda g}=\frac{5 \kappa^{2} \lambda}{16 \pi^{2}} \mu^{2}-\frac{\kappa^{2} \lambda m^{2}}{2 \pi^{2}}-\frac{5 \kappa^{4} m^{4}}{64 \pi^{2}} \\
& \beta_{y_{t} g}=\frac{\kappa^{2}}{2 \pi^{2}} y_{t} \mu^{2}, \gamma_{\phi g}=-\frac{\kappa^{2} m^{2}}{32 \pi^{2}}, \gamma_{t g}=\frac{27 \kappa^{2}}{512 \pi^{2}} \mu^{2} .
\end{aligned}
$$

\section{The potential due to gravity corrections}

We solve the RG equations for the coupling constants and $V_{\text {eff }}$, using the $\beta$ functions and anomalous dimensions due to $\mathrm{SM}^{3}$ and gravity corrections given in (8). We 
employ the threshold values of these quantities given in the literature ${ }^{5}$

$$
\begin{aligned}
& g_{y}\left(m_{t}\right)=0.45187, g_{2}\left(m_{t}\right)=0.65354, \\
& g_{3}\left(m_{t}\right)=1.1645-0.00046\left(\frac{m_{t}-173.15}{\mathrm{GeV}}\right), \\
& y_{t}\left(m_{t}\right)=0.93587+0.00557\left(\frac{m_{t}-173.15}{\mathrm{GeV}}\right)-0.00003\left(\frac{m_{H}-125}{\mathrm{GeV}}\right), \\
& \lambda\left(m_{t}\right)=0.12577+0.00205\left(\frac{m_{H}-125}{\mathrm{GeV}}\right)-0.00004\left(\frac{m_{t}-173.15}{\mathrm{GeV}}\right) .
\end{aligned}
$$

We adjust the value of $m^{2}\left(m_{t}\right)$ so that $V_{\text {eff }}(\phi)$ gives correct the vacuum, $v=$ $246 \mathrm{GeV}$.
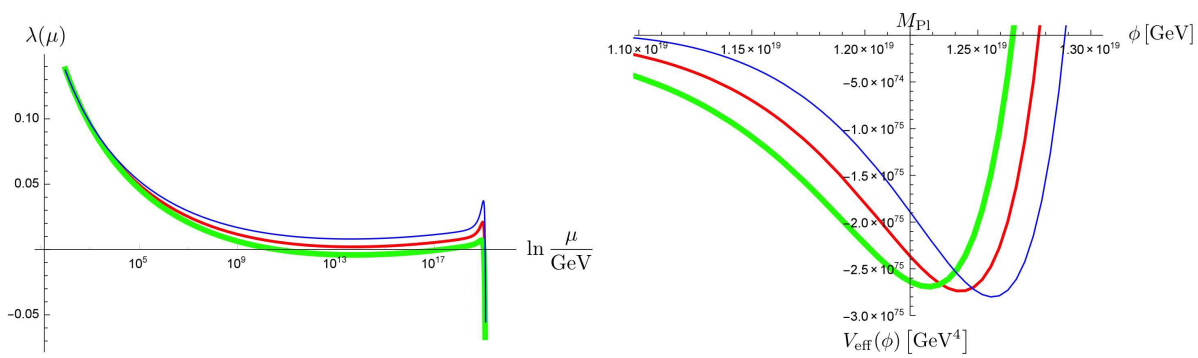

Fig. 2. RG flow of $\lambda(\mu)$ and $V_{\text {eff }}(\phi)$ at $\phi \sim M_{\mathrm{Pl}}$ These curves correspond to top mass values $m_{t}=174,173,172 \mathrm{GeV}$ in thick and color order, Green, Red, Blue.

The influence of the gravitational corrections to $\lambda(\mu)$ and $V(\phi)$ is very small, the $\lambda(\mu)$ and $V(\phi)$ are consistent with those in SM, at $\mu, \phi<(O)\left(10^{17}\right) \mathrm{GeV}$.

i) $\mu$-dependence of $\lambda$

Gravity corrections are noticeable around $\mu=\mathcal{O}\left(10^{18} \mathrm{GeV}\right)$, having the effect of increasing the value of $\lambda$ rapidly, as seen in the Fig2 2 . This behavior stops at $\mu=(0.9 \sim 1.0) \times M_{\mathrm{Pl}}$, and they start to decrease $\lambda$ sharply. $\lambda$ becomes negative at $\mu \sim M_{\mathrm{Pl}}$.

ii) $V(\phi)$ near $\phi \sim M_{\mathrm{Pl}}$

Regarding $V(\phi)$, gravitational effects begin to be noticeable at $\phi=\mathcal{O}\left(10^{18} \mathrm{GeV}\right)$, $\phi^{6}$ and $\phi^{8}$ terms become dominant. $\phi<M_{\mathrm{Pl}}, V(\phi)$ is increasing and is positive. $\phi \sim(0.8 \sim 0.9) \times M_{\mathrm{Pl}}, V(\phi)$ begins to fall. It stops falling at $\phi=1.1 \times M_{\mathrm{Pl}}$, and takes the minimum there. At $\phi \sim 10^{20} \mathrm{GeV}$ and larger, $V(\phi)$ is rapidly increasing. However, at such large values of $\phi$, more higher loop effects will be dominant, one cannot say anything reliable about graviton loop corrections.

iii) $\Lambda$ dependence of $V(\phi)$

At $\phi \sim M_{\mathrm{Pl}}$ or larger, $\phi^{6}$ and $\phi^{8}$ terms are significant but they depend on the cut-off value $\Lambda$, as shown in Fig $3 . V(\phi)$ takes the minimum for each cut-off $\Lambda=$ $1 \times M_{\mathrm{Pl}}, 2 \times M_{\mathrm{Pl}}, 3 \times M_{\mathrm{Pl}}$. One may safely say that $V_{\mathrm{eff}}(\phi)$ takes a minimum; 


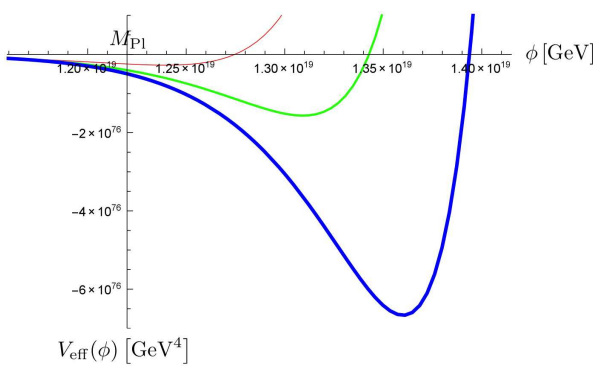

Fig. 3. $V_{\text {eff }}(\phi)$ at $\phi \sim M_{\mathrm{Pl}}, m_{H}=$ $125.09 \mathrm{GeV}, m_{t}=173.21 \mathrm{GeV}$ and $\Lambda=$ $3 M_{\mathrm{Pl}}, 2 M_{\mathrm{Pl}}, M_{\mathrm{Pl}}$ in thick order. The vertical axis is on the position of $\phi=$ $M_{\mathrm{Pl}}$.

the depth of the minimum depends strongly on the cut-off value $\Lambda$, however. The value of $\phi$ at the minimum $\left(\phi_{m}\right)$ increases proportion ally to cut-off $\Lambda$. However $\Lambda$ dependence of $\phi_{m}$ is mild, $\phi_{m}$ stays around $M_{\mathrm{Pl}}$. Hence, $V(\phi)$ takes the minimum at $\phi<\Lambda$ except the case of $\Lambda=1 \times M_{\mathrm{Pl}}$.

\section{Gravity corrections in $R^{2}$ gravity}

We have evaluated the quantum gravity corrections to Higgs potential $V(\phi)$ in the Einstein gravity in the cut-off method. The resulting $V(\phi)$ apparently depends on the cut-off $\Lambda$. It is desirable to study the gravity corrections in some UV renormalizable modified gravity, so that they have no $\Lambda$ dependence or at most mild $\Lambda$ dependence. One modest approach is to take $R^{2}$ gravity and evaluate $R^{2}$ gravity C-W corrections to $V(\phi)$. A different approach has been taken in an early work ${ }^{14}$. A certain $R^{2}$ gravity, i.e. the action of $R^{2}$ term $+\left(R_{\mu \nu}\right)^{2}$ term is known to be UV renormalizable. $\stackrel{18,19}{ } R^{2}$ term alone may not be renormalizable but the UV divergence behavior is mild, and this theory tentatively serves our purpose.

$$
S=\int d^{4} x \sqrt{-g}\left[\frac{2}{\kappa^{2}}\left(R+\alpha R^{2}\right)+\cdots\right] .
$$

In this case, graviton's propagator is $i P_{\mu \nu ; \rho \sigma}$, where

$$
P_{\mu \nu ; \rho \sigma}=\frac{\eta^{\mu \nu} \eta^{\rho \sigma}-\eta^{\mu \rho} \eta^{\nu \sigma}-\eta^{\mu \sigma} \eta^{\nu \rho}}{2 k^{2}}-\frac{1}{4} \frac{\eta^{\mu \nu} \eta^{\rho \sigma}}{k^{2}+\frac{1}{4 \alpha}} .
$$

The difference between the propagator of the $R^{2}$ gravity and the Einstein gravity is the presence or absence of the second term at (11). Due to the second term of the propagator, we get the UV finite 1-loop effects about $\phi^{6}$ term

$$
-\frac{\kappa^{2} \lambda^{2}}{20 \pi^{4}} \ln \left(4 \alpha \mu^{2}\right) \phi^{6}
$$

As expected, the cut-off $\Lambda$ does not appear. The same remark may not hold true for $\phi^{8}$ and higher terms.

\section{Conclusions}

Evaluating the quantum gravity corrections to the Higgs potential $V(\phi)$ in the Einstein gravity in the cut-off method, we have found a salient difference between 
$V(\phi)$ with and without gravity corrections in the region $\phi \gtrsim M_{\mathrm{Pl}} . V(\phi)$ with gravity corrections takes a minimum at $\phi \sim M_{\mathrm{Pl}}$, while $V(\phi)$ without gravity corrections monotonically increases. $V(\phi)$ depends on the cut-off scales, because the Einstein gravity is not renormalizable. As seen in Fig [3, $\Lambda$ dependence of the depth of the minimum of $V(\phi)$ is strong. Whereas, the value of $\phi$ at the minimum depends weakly on the value of $\Lambda$, the minimum exists regardless of the cut-off scales. We may safely guess that qualitatively due to gravity corrections, the Higgs potential takes the minimum around the $\phi \sim M_{\mathrm{Pl}}$.

There is a possibility of that Higgs potential does not depend on the cut-off scales if we consider some modified theories of gravity. In a simple case of $R^{2}$ gravity ( without $\left(R_{\mu \nu}\right)^{2}$ term), the $\phi^{6}$ term correction does not depend on $\Lambda$.

\section{Acknowledgment}

This talk(M. H.) is supported in part by funding from Shinshu University.

\section{References}

1. N. Cabibbo, L. Maiani, G. Parisi and R. Petronzio, Nucl. Phys. B 158, 295 (1979).

2. M. Spira and P. M. Zerwas, Lect. Notes Phys. 512, 161 (1998).

3. Y. Hamada, H. Kawai and K. Oda, Phys. Rev. D 87, 053009 (2013).

4. F. Bezrukov, M. Y. Kalmykov, B. A. Kniehl and M. Shaposhnikov, JHEP 1210, 140 (2012).

5. G. Degrassi, S. D. Vita, J. EliasMiro, J. R. Espinosa, G. F. giudice, G. Isidori and A. Strumia, JHEP 1208, 098 (2012).
6. D. Buttazzo,
G. Degrassi,
P. P. Giardino,
G. F. Giudice,
F. Sala, A. Salvio and A. Strumia, JHEP 1312, 089 (2013).

7. A. Andreassen, W. Frost and M.D. Schwartz, Phys. Rev. Lett. 113, 24, 241801 (2014)

8. A. V. Bednyakov, B. A. Kniehl, A. F. Pikelner and O. L. Veretin, Phys. Rev. Lett. 115, 20, $201802(2015)$.

9. F. Loebbert and J. Plefka, Mod. Phys. Lett. A 30, 34, 1550189 (2015).

10. S. Bhattacharjee and P. Majumdar, Nucl. Phys. B 885, 481 (2014).

11. N. Haba, K. Kaneta, R. Takahashi and Y. Yamaguchi, Phys. Rev. D 91, 016004 (2015).

12. Y. Abe, M. Horikoshi, T. Inami, arXiv 1602.03792

13. S. R. Coleman and E. J. Weinberg Phys. Rev. D 7, 1888 (1973).

14. L. Smolin, Phys. Lett. B 93, 95 (1980)

15. C. Ford, I. Jack and D. R. T. Jones, Nucl. Phys. B 387, 373 (1992).

16. K. G. Chetyrkin and M. F. Zoller, JHEP 1304, 091 (2013).

17. A. V. Bednyakov, A. F. Pikelner and V. N. Velizhanin, Nucl. Phys. B 875, 552 (2013).

18. K. S. Stelle, Phys. Rev. D 16, 953 (1977).

19. E. S. Fradkin and A. A. Tseytlin, Nucl. Phys. B 201, 469 (1982). 